\title{
Control issues of a fast positioner intended for deflection of a high power laser beam
}

\author{
Michat Bartyś ${ }^{*}$, Maciej Bodnicki², Karol Bagiński², Wojciech Credo $^{2}$, Leszek Wawrzyniuk ${ }^{2}$ \\ and Jakub Pełka \\ ${ }^{1}$ Institute of Automatic Control and Robotics, Warsaw University of Technology, A. Boboli 8, 02-525 \\ Warsaw, Poland \\ ${ }^{2}$ Institute of Micromechanics and Photonics, Warsaw University of Technology, A. Boboli 8, 02-525 \\ Warsaw, Poland
}

\begin{abstract}
This paper discusses some crucial aspects of control of a fast positioner intended for positioning of a mirror reflecting high power laser beam. Positioner is driven by a two linear movement piezo actuators acting on a common flexible flat spring bending it in two perpendicular directions. The reflector is fixed to this spring. Clearly, the actuator is a dynamic oscillating system. This makes control of positioner demanding. In order to overcome the effects of static nonlinearity, hysteresis and their time evolution, there were proposed two independent closed loop positioning systems each governing each own axis. The simulation investigations allowed for determining the suboptimal structure and parameters of controllers. Two different control loops were investigated and discussed. The simulation experiments shown, that the most efficient controller is able to "mask" the actuator hysteresis while introducing appropriate dynamic damping and stiffness to the controlled system. The concept of the positioner controller was described and some chosen results of laboratory experiments were presented. Finally, the assessment of the control quality is given together with some general conclusions closing the paper.
\end{abstract}

Keywords: piezoelectric actuator, fast positioning unit, hysteresis, control error, positioner, tracking error, laser beam reflector

\section{Introduction}

Since many years the piezo elements have been applied successfully for design of actuating and sensing devices. There are numerous examples of massive industrial applications of piezo elements for driving pilot valves both in electro-pneumatic as well as in electrohydraulic control valves [1-3]. Contemporary diesel and gasoline common rail injector control valves are almost dominating in design of combustion engines [2]. Despite the piezoelectric effect, the intrinsically save piezo elements are applied nowadays widely even in areas where atmospheres are prone to explosions and thus replacing traditional solenoid based valves $[1,4]$. They are applied in electro-pneumatic transducers used in final control

\footnotetext{
*Corresponding author: bartys@mchtr.pw.edu.pl

Reviewers: Maciej Berdychowski, Alžbeta Sapietová
} 
elements intended for applications in explosive zones [4]. Commonly, the piezoelectric motors are applied for driving motors and servo focus in a continuous autofocus mode of lens actuators [6-11]. Moreover, the piezo elements are widely used for measurement of acceleration, pressure, force, strain and acoustic emissions [12].

Commonly, piezo-elements are recognized as ultralow power consumption devices. For example a power barely equal to $4 \mathrm{~mW}$ is necessary to drive piezotronic proportional-minipiezo-valve [3]. Power consumption data may be wrongly interpreted particularly in the case of driving dynamic systems. Some comments to driving power in regard to positioner intended for deflection of a high power laser beam being the subject of interest of this paper is discussed later. On the other hand, piezo-elements exhibit evident static nonlinear static characteristics, hysteresis and natural resonant frequency. Additionally, all mentioned above characteristics are subject to evolutionary changes. In part, this explains why the piezoelements are used frequently for binary actuated devices. It such applications nonlinear static characteristics, hysteresis and their time evolution are unimportant. Therefore, in these applications the open loop control systems are widely deployed.

In contrast, for the continuous actuating systems, these parameters play an important role and must be taken into consideration because they seriously influence: linearity, stability, reproducibility, and variance of parameters of the system. In order to overcome these problems, a closed loop control systems are applied. The basic idea is to "mask" the drawbacks of the piezo-actuator by applying high quality feedback signal and profiting from linearization effect of the closed loop systems. The only problem is to design a proper structure and assign appropriate parameters of such controller.

The main aim of this paper is to discuss and propose systematically a structure of a control system intended for driving laser beam deflecting mirror [13]. The mirror actuating system consists of two piezo actuators driven by two independent control units. The overview of the mechanical construction of the mirror actuating assembly is shown in Fig. 1.

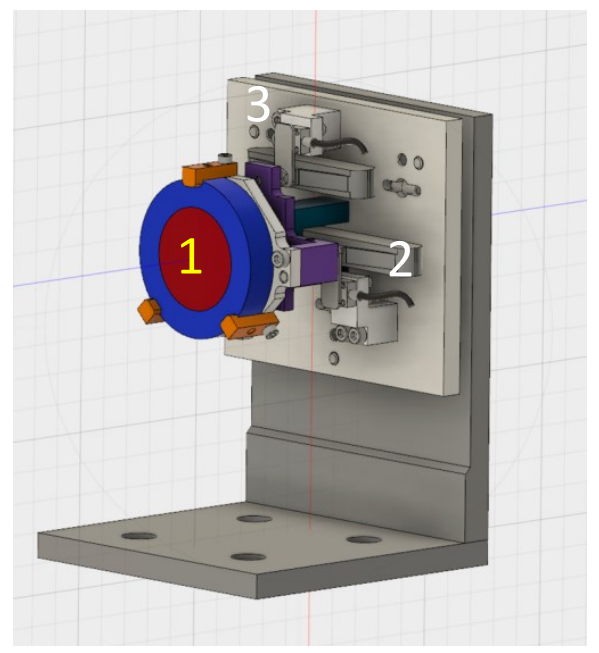

Fig. 1. A view of the mechanical construction of the laser beam deflection mirror. Notions: 1 - mirror; 2,3 - piezo-actuators with tensometer based displacement transducers in $x$ and $y$ direction

Principally, the mirror actuating assembly plays a role of a positioning system. Positioner is driven by a two linear movement piezo-actuators acting on a common flexible flat spring bending it in two perpendicular directions $x$ and $y$. The reflector is fixed to this spring. Clearly, the actuator is a dynamic oscillating system. This makes control of positioner demanding. The angle of mirror reflection in each axis is spanned over $\pm 3.5 \mathrm{mrad}$. The dynamic requirements are set very stringent. The limit frequency band was set to $1000 \mathrm{~Hz}$. 
This is the reason why further in the text we will refer to a fast actuator. In order to simplify the design of a control system, there were proposed and applied two independent closed loop positioning systems each governing each own axis. This allows for linearization of the angular movement as well as or for isolation of mechanical mirror coupling effect caused by mutual interactions of piezo-actuators acting on the same flat spring. The simulation investigations allowed for determining the suboptimal structure and parameters of controllers. Two different control loops were investigated and discussed. The simulation experiments shown, that the most efficient controller is able to "mask" the actuator hysteresis while introducing appropriate dynamic damping and stiffness to the controlled system.

The paper contributes to the applied control of the fast piezo-actuating systems. It delivers some useful and experimentally proven parametrized structure of the control system.

The paper is structured in a following way. In the introductory part, a control problem is highlighted. Some thoughts and remarks concerning trade-off between mechanical and electrical power of piezo actuators in context of control reachability are given in Sec. 2. Section 3 presents some results of experimental identification of the system being controlled and explains why the open loop control system is infeasible. Sect. 4 brings some results of simulations, while in Sect. 5, the chosen results of implementation of the digital control systems are shown. Final remarks finalize the paper.

\section{Electrical versus mechanical power}

\subsection{Electrical power}

Without any doubts, piezo-elements are happy to apply whenever a low power in a steady state is needed for a controlled system. This may not be true if the actuator will be used for driving high frequency systems. In a low frequency band, the piezo element is an electrical device which electrical properties can be pretty well and sufficiently represented by an equivalent ideal capacitive element. In fact, for a few $\mathrm{kHz}$ band, the inductance and resistance of the actuator might be neglected. The electrical capacity of the piezoelectric devices depends of their construction, shape, temperature and physical features of piezo material. The capacity of the piezo elements may range in a span starting from a few $\mathrm{nF}$ up to the hundreds of $\mu \mathrm{F}$. For applications in explosive areas the capacity of the piezo-element is typically under $100 \mathrm{nF}$. In contrast, the capacitance of the piezo element used in high power laser beam reflector equals circa $4.1 \mu \mathrm{F}$. While the piezo element represents almost pure capacitive load to the alternating voltage source, the average real power theoretically equals zero. Therefore, the only reactive power should be considered when discussing power demands of the actuator.

In an alternating current circuit, the instantaneous power $p_{i}$ for sinusoidal excitation is equal to the sum of real $p_{r}$ and reactive power $p_{x}$,

$$
p_{i}=p_{r}+p_{x} \text {. }
$$

The real power equals to:

$$
p_{r}=U_{p} \cdot I_{p} \frac{1-\cos (2 \omega t)}{2} \cos (\varphi),
$$

where: $U_{p}$ is the peak voltage, $I_{p}$ is the peak current, $\varphi$ - the phase angle between the current and voltage waves, $\omega=2 \pi f, f$ - frequency of sine wave, $t$ - time.

As far as the piezo-element is assumed as an ideal capacitor, the average and peak real powers equal zero because phase shift angle $\varphi=\pi / 2$. Therefore, in this case, the only reactive power will circulate between the excitation voltage source and capacitance of the piezoelement. If the real systems, the power loss due to internal ohmic resistance of the excitation 
source combined with connecting wires resistance can't be neglected. Thus, the peak real power loss is different from zero and is equal to:

$$
p_{r}=I_{p}^{2} R_{e}
$$

where: $R_{e}=R_{i}+R_{c}$ is the sum of the internal resistance of the excitation source $R_{i}$ and resistance of wires connecting excitation source with the piezo-element.

The real power loss may not be neglected particularly for higher frequencies of excitation signal, where the peak values of the current are significant (see Fig. 2). The reactive power equals to:

$$
p_{x}=U_{p} \cdot I_{p} \frac{\sin (2 \omega t)}{2} \sin (\varphi) .
$$

It is clear that for $\varphi=\pi / 2$ the average reactive power equals zero too. However, the peak reactive power $p_{p x}$ is different from zero. Substituting $I_{p}$ in (4) by $I_{p}=2 \pi f C U_{p}$, where $C$ is capacitance of the piezo-element, we obtain:

$$
p_{p x}=\pi f C U_{p}^{2}
$$

As can be easily seen from (5), the electrical reactive power needed for driving piezo-actuator is proportional to its capacitance and driving frequency. The peak reactive power and peak reactive current for a piezo-element applied in the laser beam driver positioner are shown respectively in Figs. 2 and 3. As can be easily seen, the theoretical reactive driving power may be quite serious.

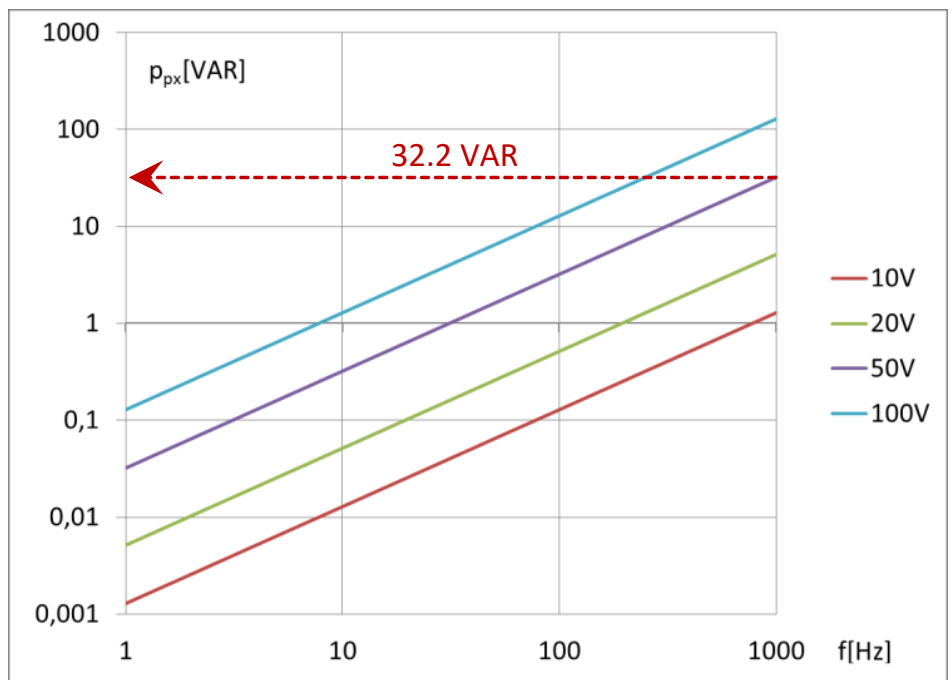

Fig. 2. Maximum reactive power versus frequency for a piezo-element actuator APA150M [5]. The maximal capacitance of the piezo-element equals $4.1 \mu \mathrm{F}$. Chart parameter: peak excitation voltage $U_{p}$ 


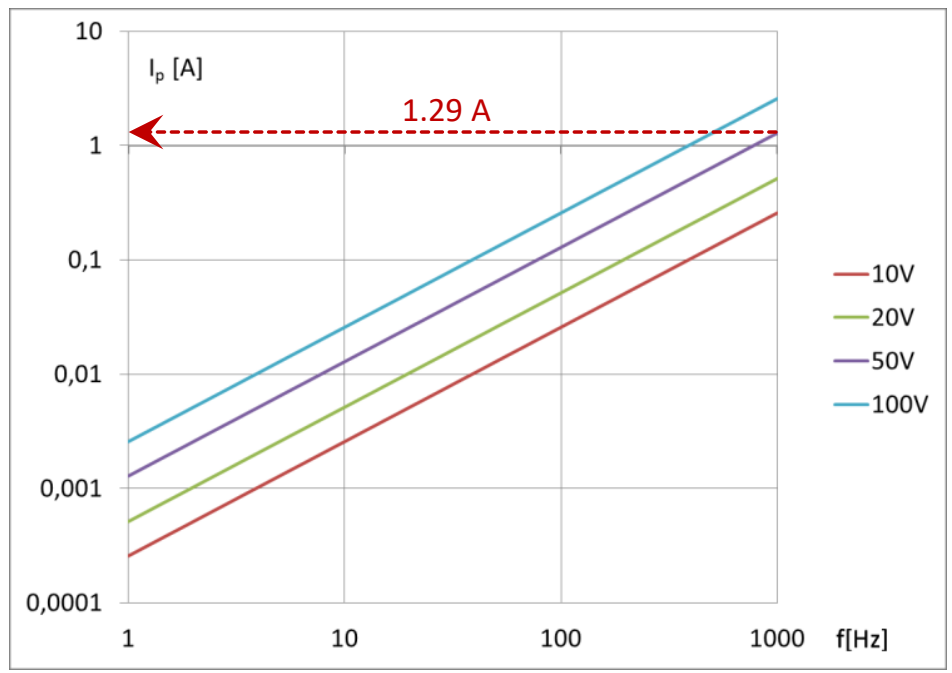

Fig. 3. Maximum reactive current versus frequency for a piezo actuator APA150M. The maximal capacitance of piezo element equals $4.1 \mu \mathrm{F}$. Chart parameter: peak excitation voltage $U_{p}$

\subsection{Mechanical power}

The electrical power is transformed by the actuator into mechanical one. Let us suppose, that actuator swings up and down harmonically with the frequency $f$ and amplitude $A$. The displacement of actuator is opposed by a static as well as dynamic load. Let the equivalent stiffness of the piezo actuator be $k_{x}$. Additionally, assume that the $k_{a}=$ const. For simplicity, let us neglect gravity force. Hence, the change of linear displacement $x$ in a steady state of unloaded actuator will be proportional to the applied force $F_{x}$ induced by voltage excitation $U$ of the piezo-actuator:

$$
F_{x}=k_{x} \cdot x
$$

Therefore, the mechanical $E_{a}$ energy stored in actuator in steady state is:

$$
E_{x}=k_{x} \cdot x^{2} .
$$

Now, let us assume that actuator is swinging harmonically.

$$
x=X \cdot \sin (\omega t),
$$

where: $X$-amplitude of harmonic movement

Further, the velocity and acceleration of the movement are respectively as follows:

$$
\begin{aligned}
& \dot{x}=X \omega \cdot \cos (\omega t), \\
& \ddot{x}=-X \omega^{2} \cdot \sin (\omega t) .
\end{aligned}
$$

From (9) and (10) the maximum velocity equals $\dot{\hat{x}}=X \omega$ while maximal acceleration $\ddot{\hat{x}}=X \omega^{2}$. Let us now assume that piezo-actuator will drive a constant mass $m$. According to the second Newton's law of motion, the rate of change of momentum of a body is directly proportional to the force applied in the direction of the applied force, Hence, after substituting (6) by (10) we obtain:

$$
F_{x}=-m X \omega^{2} \cdot \sin (\omega t) .
$$

The mechanical power $p_{m}$ is proportional to the applied force and velocity. Hence:

$$
p_{m}=-\frac{1}{2} m X^{2} \omega^{3} \cdot \sin (2 \omega t)
$$


Therefore, maximum absolute value of mechanical power: $p_{m}=0.5 \mathrm{~m} X^{2} \omega^{3}$. Let us estimate maximum acceleration and mechanical power that must be applied to move harmonically the mass $m=100 \mathrm{~g}$ within frequency range $0 . .1000 \mathrm{~Hz}$. The results are shown in Figs. 4 and 5.

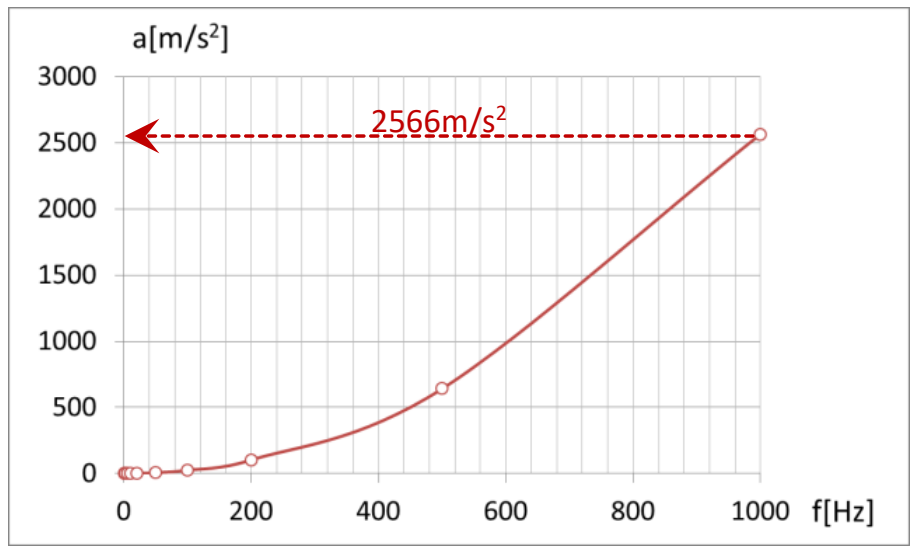

Fig. 4. Maximum acceleration versus frequency for a harmonic movement of a mass $m=0.1 \mathrm{~kg}$ with amplitude $X=65 \mu \mathrm{m}$

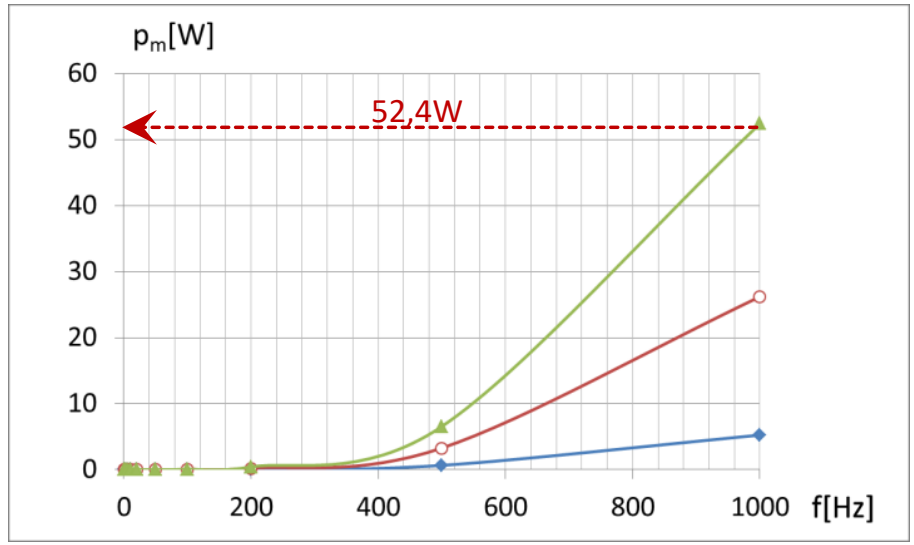

Fig. 5. Maximum mechanical driving power of the constant-mass $m$ versus frequency for a harmonic movement with amplitude: $X=65 \mu \mathrm{m}$. Notions: blue line $(m=0.01 \mathrm{~kg})$, red line $(m=0.05 \mathrm{~kg})$, green line $(m=0.1 \mathrm{~kg})$

Further we will assume piezo actuator as a linear time-invariant system (LTI). Let us now discuss property of reachability of control for this system.

Example: The mirror positioning system presented in introductory section having mass $m=89,9 \mathrm{~g}$, amplitude $X=110 \mu \mathrm{m}$, capacitance of piezo actuator $C=4.1 \mu \mathrm{F}$, swinging harmonically with frequency $f=1000 \mathrm{~Hz}$ and driven by an alternating voltage source with $U_{p}=85 \mathrm{~V}$ amplitude, exhibits the demand on maximal mechanical power (12) $p_{m}=47,1 \mathrm{~W}$ while the electrical reactive power (5) that can be delivered to actuator equals $p_{p x}=93,1 \mathrm{VAR}$. Hence: $p_{p x}>p_{m}$. This clearly shows that the control system will have the property of reachability in the given frequency range even if electrical to mechanical conversion effectiveness will be as low as $50 \%$. 


\section{Experimental identification of piezo-actuator}

Doubtless, the identification of the properties and parameters of the controlled system is considered as fundamental for proper design of the control system. There were developed a numerous system identification approaches suitable for application in the control practice. Fortunately, in case of high power laser beam reflector, the identification of system under control could be performed experimentally. Because of restricted volume of this paper, the laboratory test rig which has been used for experimental identification of piezo-actuator features will not be described here. Details can be found in [13]. Principally static as well as dynamic properties of the investigated actuator have been taken from experiments. Main results which have been obtained are shown and discussed in consecutive subsections.

\subsection{Steady state characteristics of the piezo-actuator}

According to definition, the continuous system or a process is in a steady state if all derivatives of all its states or signals with respect to time tend to zero. Steady state characteristic is a powerful tool for discriminating between linear and nonlinear systems. From the technical point of view, the steady state characteristics allows for estimating some important and characteristic features of the system e.g. linearity, reproducibility and hysteresis. As a rule, the actuators are used in automation processes as final control elements. The final control element transform low power output of controller into the mechanic power needed for direct driving plant facilities.

Each of both piezo actuators considered in this paper may be presented in the black box schematics as shown in Fig. 6. For simplicity the only one ( $x$ axis) is shown. In fact, Fig. 6 presents very general concept of the closed loop system.

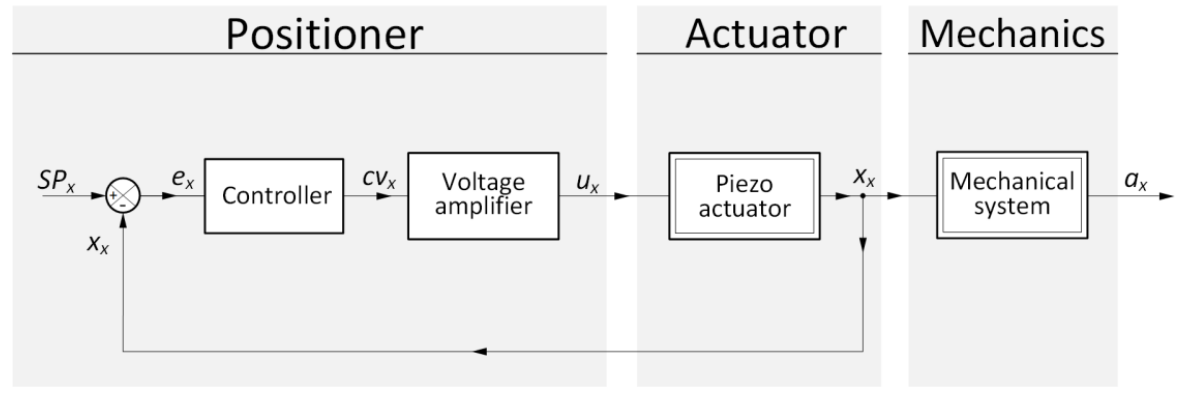

Fig. 6. Block schematics of the piezo actuator closed loop control system. Notions: $u_{x}$ - piezo element excitation voltage; $x_{x}$ - linear displacement of the piezo actuator; $a_{x}$ - angular displacement of the mirror of piezo actuator; $S P_{x}$ - displacement setpoint value; $e_{x}$ - control error

The external setpoint control signal $S P_{x}$ drives the piezo actuator by means of voltage and power amplifier. Resulting piezo actuator displacement $x_{x}$ is converted into angular displacement $\alpha_{x}$ of the actuator's mirror. Unfortunately, the angular displacement $\alpha_{x}$ is not subject of measurement. This means that the angular position of the mirror cannot be controlled directly. The mirror is positioned on the flat spring which is tensed and moved by the actuator movement $x_{x}$. The mechanical translation between actuator movement $x_{x}$ and angular displacement $\alpha_{x}$ is very complex and nonlinear. Moreover, as experiments show, the angular displacement $\alpha_{x}$ is weakly depending on the angular displacement $\alpha_{y}$ and vice versa. Therefore, the correction of the angular position in steady state might be theoretically possible in open loop control loop by means of two-dimensional interpolation. This however, is highly impractical. Therefore, feasible is to apply separate closed loop control systems 
controlling each axis independently. Each of control loop has to linearize nonlinear steady state characteristics of the piezo-actuator as well as to mask hysteresis of the piezo actuator.

Let us now discuss briefly the application of an open loop control system for controlling piezo actuator. For the sake of the simplicity let us limit our considerations to only one $x$ axis. Fig. 7. shows the normalized reproducibility of the displacement of the actuator versus normalized control signal. This experimental characteristic exhibits clearly very high reproducibility of the actuator. This characteristic has been taken by constant ambient temperature and unidirectional change of control signal.

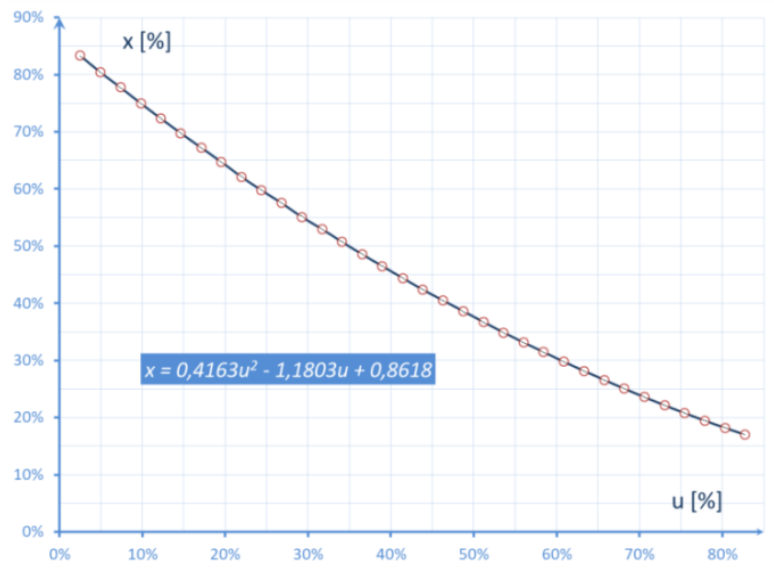

Fig. 7. Experimental normalized controller output voltage $u_{x}$ versus normalized displacement of actuator $x_{x}$

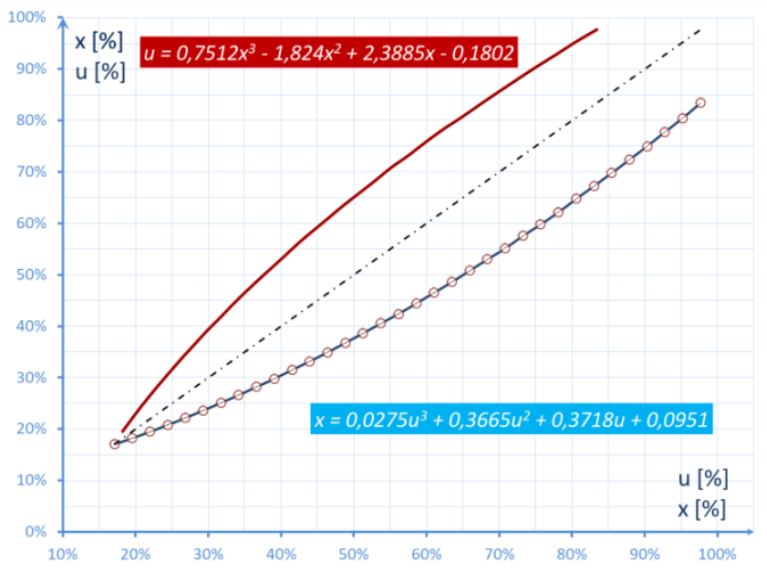

Fig. 8. Illustration of construction of inverse function (red line) for compensating nonlinearity of controller output $u_{x}$ by given desired actuator displacement $x_{x}$

This characteristic can be approximated by the third order polynomial:

$$
x=0,0275 u^{3}+0.3665 u^{2}+0,3418 u+0,0951 .
$$

Hence, the compensation of the nonlinearity of this characteristic can be easily done by assignment of the inverse function $u=f(x)$. which is symmetrical in respect to straight line $u=x$. The inverse function to (13) is as follows:

$$
u=0,7512 x^{3}-1.824 x^{2}+2,3885 x-0,1802 .
$$


The inverse function can be easily implemented in controller by means of equation (14) or by application of a look-up table technique. Particularly, this second approach makes control algorithm running extremely fast.

There are numerous sources of nonlinearities in piezo-actuator. Moreover, this nonlinearities are depending on other physical quantities e.g. temperature. Therefore, the accurate positioning in open control loop is not reasonable. The open control systems require at least keeping good reproducibility of the steady state characteristics. This is a case for the piezo-actuator type APA150M [5] (Fig. 7). However, the naked eye look in the Fig. 8 shows evident nonlinearity of the steady state characteristics. Fortunately, as far as this characteristic is reproducible, the open loop control system may compensate this nonlinearity by applying reverse characteristics.

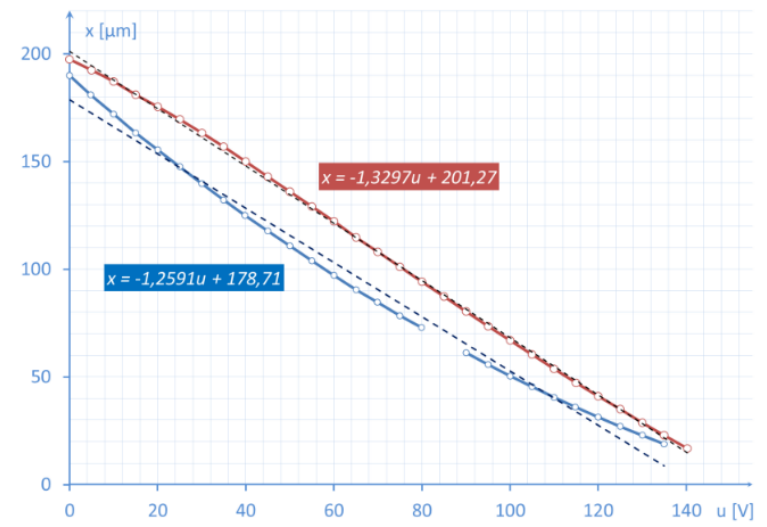

Fig. 9. Hysteresis of the unloaded piezoactuator type APA150M taken from experiments. The maximal hysteresis equals $14,0 \%$

Unfortunately, the steady state characteristic exhibits hysteresis ca $14 \%$ visible in Fig. 9. Thus, clearly, the open loop system does not guarantee obtaining demanded values of control quality factors. Therefore, piezo-actuator type APA150M should unconditionally be governed by a closed loop system. This may also bring additional profits particularly by suppressing influence of temperature and changes of actuator steady state characteristics.

In fact, the steady state characteristics of the actuator are nonlinear and may be narrowed down by a backlash system. This allows for simplify simulation experiments of the control system. Mechanical construction of the laser beam deflector for both $x$ and $y$ axis is slightly different. The deflections rates for both axes are similar. As experiments showed, the $x$-axis is rated at $127,5 \mu \mathrm{m} / 100 \mathrm{~V}$ while equal $130,55 \mu \mathrm{m} / 100 \mathrm{~V}$ for y axis. By appropriate settings of closed loop controllers there is possible to adjust and keep constant both rates.

Given above considerations lead to the conclusion, that application of the closed loop system driving laser beam deflector is undisputable. However, the problem is how to solve and implement appropriate control system.

Obviously, the hysteresis envelopes vary with the amplitude and offset of the excitation voltage. Here, the inner hysteresis loops may be visible in the steady state characteristics of the piezo actuator. Due to the complexity of this phenomenon, this effect will not be further discussed nor embedded into the model. Therefore, the worst case hysteresis loop will be exploited in order to look for the worst case. The hysteresis is assumed to be a backlash with a play limited by

$$
x_{x}=x_{x h} \cdot \frac{1}{2}\left[1+\operatorname{sgn}\left(\frac{d u_{x}}{d t}\right)\right]+x_{x l} \cdot \frac{1}{2}\left[1-\operatorname{sgn}\left(\frac{d u_{x}}{d t}\right)\right] .
$$

The hysteresis of displacement $x_{y}$ of $y$-axis piezo actuator element is modelled analogously. 


\section{Control problem}

The control system for high frequency laser beam deflector unit should response to some crucial technical requirements while maintaining standard control loop requirements such as stability and sufficient amplitude and phase margins. The most important requirements are demanding and are listed below:

- teady state positioning error should not exceed $0.1 \%$,

- high frequency limit of the amplitude characteristic should be at least $1000 \mathrm{~Hz}$,

- phase margin should be at least $\pi / 4$ and amplitude margin should be at least $3 \mathrm{~dB}$.

While the control system is nonlinear, it is feasible to follow up experimentally verified procedure of simulation of control system. This may narrow down expected dynamic and static behavior of the controlled system. Because of limited space of this paper we omit a long way process of obtaining simulation model of the piezo-actuator, determination its parameters and optimizing process of control system structure. We show only some final results of simulations in matlab-simulink environment as well as chosen results of implementation of the controller.

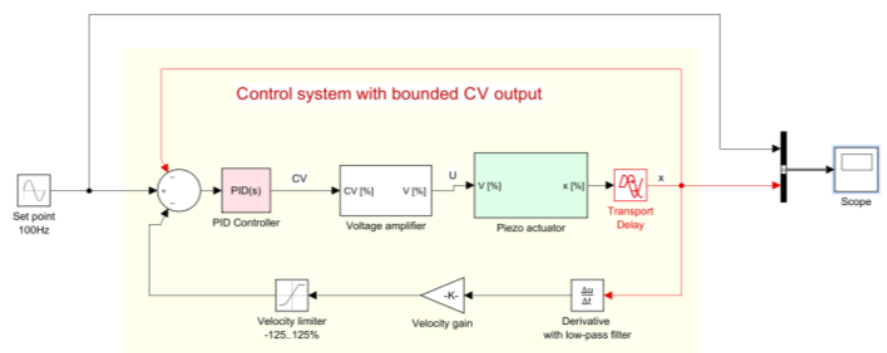

Fig. 10. Closed loop control system with bounded output and bounded velocity feedback

Final model structure of the control system intended for fast positioning of the angular position of the reflector of laser beam is depicted in Fig. 10. Additionally, a "look under the mask" of piezo-actuator model is shown in Fig. 11. As easily can be seen from Fig. 11, the piezo-actuator model reflects its static nonlinearity, backlash as well as linear dynamics. Additionally, the delay $(\tau=300 \mu \mathrm{s})$ was introduced in order to reflect the time delay between excitation voltage applied to piezo-actuator and piezoactuator's response. This delay represents mainly the dynamics of the internal control system of voltage amplifier applied for experiments. Oscillations caused by introduction of delay (Fig. 12) clearly show the need of taking care for minimizing delays in the system either in main or feedback loop.

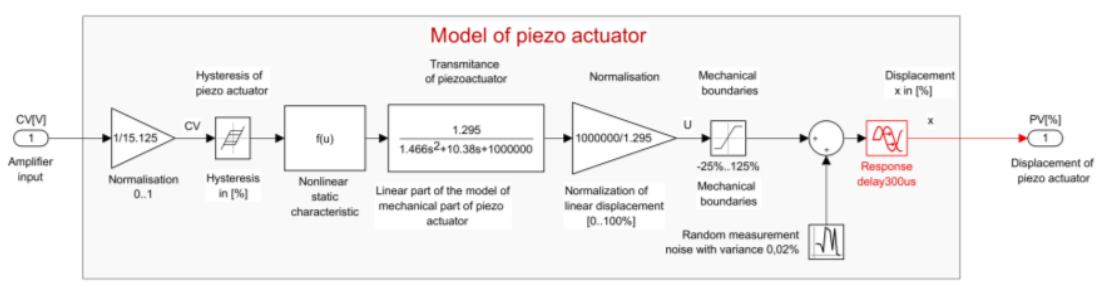

Fig. 11. Block diagram of piezo actuator model 


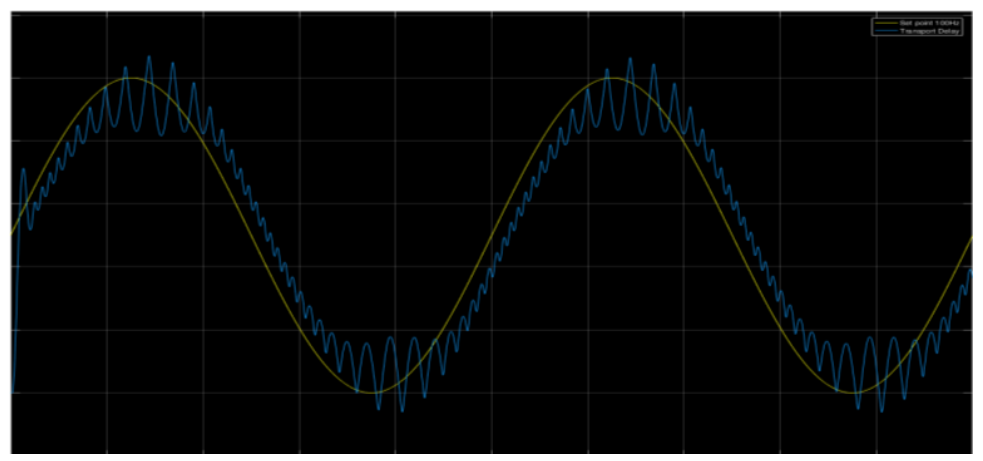

Fig. 12. Influence of delay system ( $\tau=300 \mu \mathrm{s})$ reflecting time delay between excitation voltage applied to piezoactuator and piezoactuator's response

The final control system structure (Fig. 11) was developed by means of trial-and-error method. As simulation experiments show, in order to guarantee oscillation free displacement of the mirror the maximal delay in control system should not exceed $50 \mu \mathrm{s}$.

\subsection{Implementation of control loop}

According to Nyquist rate law, the minimum controller sampling frequency should not be less than twice the bandwidth. This is of course a result of theoretical considerations. In control practice, this frequency should is at least 10 to 1000 times higher. This is highly demanding requirement particular if the expected bandwidth is relatively broad. In case of considered piezo-actuators systems, the feasible controller sampling rate should not be less than $10,000[1 / \mathrm{s}]$. In other words the controller sampling time should be not greater than $100 \mu \mathrm{s}$. It is obvious that sampling time introduces inherent delay to the control loop. This may have serious influence on system stability. As deep-in investigations showed, the maximum sampling time should not be higher than $50 \mu \mathrm{s}$.

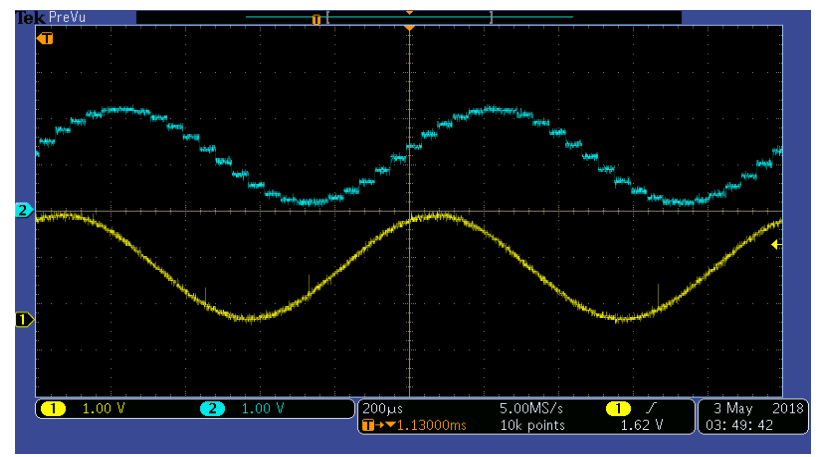

Fig. 13. An example of sine wave tracking by $P$ controller with velocity feedback. Parameters: P controller gain: $k_{p}=15$; Velocity gain: $k_{v}=2$; wave frequency $f=1000 \mathrm{~Hz}$

A digital controller reflecting control structure shown in Fig. 10 was implemented by means of ultralow power microcontroller MSP440FG4619 running with $4.9142 \mathrm{MHz}$ clock frequency. This microcontroller is equipped with embedded 12-bit resolution, successive approximation analog-to-digital converter with $5 \mu$ s conversion time and 12 bit digital-toanalog converter with maximal full scale settling time equal to $15 \mu \mathrm{s}$.

In order to obtain maximal sampling rate of the controller, the code of control structure was implemented in assembler with exhaustive usage of one cycle register-to-register 
operand addressing modes. A different PID controller structures were implemented and tested. Best results were obtained by means of PI controller with bounded output and velocity feedback. Maximal sampling time of this controller equals $28.6 \mu$ s. In contrast, sampling time equal to $15.5 \mu \mathrm{s}$ was get for $\mathrm{P}$ action controller. However, $\mathrm{P}$ action controller does not guarantee zero control error in steady state. Exemplary snap shot from the oscilloscope illustrating tracking of the $1000 \mathrm{~Hz}$ sine wave setpoint is shown in Fig. 12. Phase shift as well as discretization effect are well recognized in this case. In order to avoid this effect, the analog controller is considered instead of digital.

\section{Final remarks}

The results of theoretical discussion contrasted with the results of performed simulations and combined with the results of experimental investigations shown that the best and most efficient solution is to apply a state space feedback controller. This controller allows for increasing damping ratio and shifting eigen frequency far away while suppressing sensitivity to a high frequency noise. The simulation experiments shown, that the most efficient controller is able to "mask" the actuator hysteresis while introducing appropriate dynamic damping and stiffness to the controlled system.

The implementation of reasonable and feasible controller structure for a fast mirror positioning system should be evaluated as a challenging task. Fortunately, the conducted experiments show, that this task is achievable.

Results of experiments show evident advantages and disadvantages of digital control in context of performed task. Therefore, in the near future it is planned implementation of the analog controller which structure and parameters will be controlled digitally. It is expected to achieve lower phase shift as well as better smoothness of the control signal, particularly in the range of higher frequency band limit.

This paper has been prepared under financial support of the Polish National Centre for Research and Development, grant No DOB-1-6/1/PS/2014.

\section{References}

1. http://www.hoerbiger.com (Piezo-pneumatic control valves)

2. http://www.bosch.co.jp/tms2015/en/products/pdf/DS_ProductDataSheet_CRS3-27_EN

3. https://www.yumpu.com/en/document/view/44456368/proportional-mini-piezo-valvespiezotronic-2-2-630

4. http://www.aplisens.com.pl/produkty-ustawniki-pozycyjne.html

5. https://www.cedrat-technologies.com

6. Canon, EF Lens Work III, The Eyes of EOS. Canon Inc. (2004)

7. V. Lavrinenko, M. Nekrasov, Electrical motor. Pat. USSR 217509 (1965)

8. Vishnevsky, et al., Piezoelectric motor structures, U.S. Pat. 4019073 (1977)

9. Vasiliev, et al., Piezoelectrically driven torsional vibr. motor. U.S. Pat. 4210837 (1980)

10. A. Adams, The Camera. Little, Brown and Co. (1980)

11. D. A. Kerr, Principle of the Split Image Focusing Aid and the Phase Comparison Autofocus Detector in Single Lens Reflect Cameras.

12. G. Gautschi, Piezoelectric Sensorics: Force, Strain, Pressure, Acceleration and Acoustic Emission Sensors, Materials and Amplifiers. Springer (2002).

13. M. Bodnicki, W. Credo, L. Wawrzyniuk, K. Bagiński, M. Bartyś, Measuring system for testing of the drive of the scanning mirror. Conference on MMS, Rydzyna (2018). 